\title{
BMJ Global Health Impact of the US Food and Drug Administration registration of antiretroviral drugs on global access to HIV treatment
}

\author{
Harinder Singh Chahal, ${ }^{1}$ Peter Capella, ${ }^{2}$ Ryan Presto, ${ }^{3}$ Jeffrey S Murray, ${ }^{4}$ \\ Martin Shimer, ${ }^{3}$ Mary Lou Valdez, ${ }^{5}$ Peter G Lurie ${ }^{1}$
}

To cite: Chahal HS, Capella P, Presto R, et al. Impact of the US Food and Drug Administration registration of antiretroviral drugs on global access to HIV treatment. BMJ Glob Health 2018;3:e000651. doi:10.1136/ bmjgh-2017-000651

Handling editor Stephanie M Topp

- Additional material is published online only. To view, please visit the journal online (http://dx.doi.org/10.1136/ bmjgh-2017-000651).

Received 17 November 2017 Revised 28 February 2018 Accepted 1 March 2018
Check for updates

For numbered affiliations see end of article.

\section{Correspondence to} Dr Harinder Singh Chahal, US Food and Drug Administration harinder.chahal@fda.hhs.gov

\section{ABSTRACT}

Background Since 2004, the US Food and Drug Administration's (USFDA) dedicated drug review process in support of President's Emergency Plan for AIDS Relief (PEPFAR) has made safe, effective and quality antiretrovirals (ARVs) available for millions of patients. Furthermore, the WHO and Global Fund to Fight AIDS, Tuberculosis and Malaria (Global Fund) can add the USFDA-reviewed products to their respective formularies, through a novel process of 'one-way reliance'. We assessed the number of ARVs made available through WHO and Global Fund based on the USFDA review. Methods We conducted a cross-sectional study of all the USFDA-reviewed PEPFAR drugs between 1 December 2014 and 20 March 2017 to determine 1) the percentage that are included on the WHO and Global Fund formularies; 2) the number of the USFDA ARVs supporting the WHO HIV treatment guidelines, and their uptake by WHO and Global Fund and 3) time between the USFDA review and WHO review of the same ARVs.

Findings Overall, 91\% (204/224) of the USFDA products appeared on either the WHO/Prequalification of Medicines Programme (PQP) or the Global Fund ARV lists. Fortyfive per cent (100/224) and 83\% (184/224) appear on WHO/PQP and Global Fund formularies through one-way reliance, respectively. Forty-one per cent (91/224) of the USFDA products support the WHO-preferred first-line HIV treatment options. Of these 91 products, $38 \%$ and $85 \%$ of products were adopted by WHO/PQP and Global Fund through one-way reliance, respectively. Sixty-six products that were fully reviewed and registered by WHO (vs oneway reliance) had also undergone the USFDA review; 46 of these were registered by WHO after the USFDA review was complete (median delay of 559 days (IQR 233-798 days)). Conclusions The USFDA's PEPFAR process is making safe and effective ARVs available worldwide, in part because the major global ARV procurement organisations rely on the USFDA registration as proof of quality. There is room for improved information sharing and collaboration to reduce duplication of effort, save resources and further expedite access to ARVs.

\section{INTRODUCTION}

Reliance on the regulatory actions of established stringent regulatory authorities

\section{Key questions}

What is already known?

- Since 2004, the US Food and Drug Administration (USFDA) has reviewed over 250 non-brand antiretrovirals (ARVs) for use in resourceconstrained settings to provide significant costsavings and increased access.

- The USFDA-reviewed ARVs are used to treat $>11.5$ million patients with HIV globally through the US President's Emergency Plan for AIDS Relief (PEPFAR)

- WHO and the Global Fund may use, through one-way reliance, ARVs reviewed by the USFDA for the PEPFAR programme to enhance their own formularies of quality-assured drugs, which can increase procurement and access.

\section{What are the new findings?}

- This study quantified the impact of the USFDAreviewed ARVs for PEPFAR on the WHO and the Global Fund formularies.

- A substantial number of the USFDA-reviewed ARVs are used either by WHO (45\%) and the Global Fund (83\%) through one-way reliance, amplifying the downstream effects of the USFDA ARVs.

- Reviews were duplicated for $30 \%$ of the ARVs by the USFDA and WHO.

\section{What do the new findings imply?}

- The USFDA-reviewed drugs for PEPFAR impact global quality assurance mechanisms and procurement to increase the availability of ARVs in high-prevalence, low-resource countries.

- There is room for improved information sharing and collaboration between the USFDA, WHO and manufacturers to reduce duplication of effort, save resources and further expedite access to ARVs.

(SRAs) is gaining global momentum as a novel approach to improve timely access to essential, life-saving medicines while saving scarce regulatory resources. ${ }^{1-4}$ Under the concept of 'reliance', regulatory agencies in 
resource-constrained settings and entities responsible for procurement may rely on the work already done by SRAs to ensure a source of quality-assured health products. ${ }^{3-5}$ Such reliance can enhance regulatory efficiency and contain costs by preventing duplication of efforts and expenditure of limited resources. ${ }^{5-8}$

The US Food and Drug Administration's (USFDA) registration of antiretroviral drugs (ARVs) through its President's Emergency Plan for AIDS Relief (PEPFAR) process is a major source of quality assurance and reliance for global programmes. The term 'registration' in this context means drugs approved or tentatively approved by the USFDA; this is not to be confused with the registration requirement of manufacturing facilities and drug products listing that is required for all drugs distributed in the USA. Although another quality assurance mechanism, WHO's Prequalification of Medicines Programme (WHO/PQP), was available in 2004, US Department of State, the entity responsible for managing PEPFAR, decided that any ARVs purchased with the US government funds would meet the same safety, efficacy and quality standards as those sold in the USA. ${ }^{9} 10$ Thus, the US government opted to use the USFDA as the quality assurance mechanism instead of WHO/PQP. ${ }^{910}$

Since 2004, in support of PEPFAR, the USFDA has used a dedicated process to register ARVs to fight HIV globally. ${ }^{11}$ Under this process, the USFDA can either fully approve a product or issue a tentative approval for a product that may not yet be fully approved in the USA due to patents or market exclusivities, but which meets all safety, efficacy and quality requirements. ${ }^{1}$ This process is used to register non-branded versions of ARVs already available in the USA. ${ }^{1}$ Some of the products are new combinations or formulations of existing branded (ie, innovator) drugs, for example, two-drug or threedrug combinations, or paediatric formulations or doses, while others are copies of existing brand name drugs. ${ }^{1}$ Although drug costs are not a factor in the USFDA review or decisions, these non-branded drugs tend to be priced lower than the innovator products, resulting in cost-savings. ${ }^{12}$ Such products are placed on a publicly available list and can be procured abroad under PEPFAR, even as domestic sales are precluded. ${ }^{1}$ The original purpose of the dedicated process was to support PEPFAR, the largest US government single-disease-focused programme for foreign aid, which delivers testing, prevention and treatment to millions of patients with HIV globally. ${ }^{13}$ The US law permits PEPFAR to purchase ARVs with the US government funds that have been quality-assured by the USFDA. ${ }^{14}$

In addition to PEPFAR, other global programmes such as the WHO/PQP and the Global Fund to Fight AIDS, Tuberculosis and Malaria (Global Fund) have come to use the USFDA-certified PEPFAR ARVs to guide procurement of quality-assured products. ${ }^{415} 16$ This is accomplished through one-way recognition or reliance, under which WHO and Global Fund recognise that a review by the USFDA (or another SRA) is sufficient for quality assurance and accepts the USFDA-registered ARV for their own lists without additional review. In this way, the USFDA-registered ARVs support procurement through non-PEPFAR procurement mechanisms, regardless of funding source.

WHO/PQP, a multilateral programme, was established in 2001 to conduct quality assurance of HIV, tuberculosis and malaria drugs, and has subsequently expanded to include other medicines. ${ }^{17}$ It serves as a global quality assurance mechanism to assist international finance mechanisms and WHO's member states in purchasing trusted, low-cost medicines. ${ }^{910}$ Although WHO itself is not a regulatory entity, the WHO prequalification review is akin to that of the USFDA, in which WHO reviews drug applications for safety, efficacy and quality, and decides whether the product meets its criteria for quality assurance. ${ }^{9}$ As of January 2018, WHO/PQP has registered about 300 ARV products, contributing to a substantial reduction in ARV costs and increasing access to affordable life-saving treatments. ${ }^{9}{ }^{18}$ As with the USFDA, WHO/ PQP maintains a consolidated list of ARVs that the United Nations agencies (eg, Joint United Nations Programme on HIV and AIDS and United Nations Children's Fund) and financing organisations such as UNITAID and the Global Fund may use to guide procurement, regardless of funding source, for treatment programmes. ${ }^{417}$ In addition to procurement entities, Medicines Patent Pool, a non-profit that works to expedite access to essential drugs in resource-constrained settings by providing a framework in which innovator companies and generic manufacturers can share certain intellectual property rights, requires the generic drug makers to attain either WHO prequalification or SRA registration (eg, USFDA) ${ }^{19}$

The WHO list of quality-assured ARVs is generally referred to as the 'WHO/PQP list'. An ARV may be added to the WHO/PQP list in two ways: 1) through WHO's own full prequalification regulatory review of selected medicines leading to WHO approval or 2) with permission or at the request of the drug manufacturer, through one-way recognition of drugs registered by specified SRAs (eg, Health Canada, European Medicines Agency and USFDA). ${ }^{15} 17$ Drugs added through one-way recognition are not considered 'WHO prequalified'. Rather, such drugs are added to the consolidated $\mathrm{WHO} / \mathrm{PQP}$ list to increase visibility and promote use of SRA quality-assured drugs.

The Global Fund, established in 2002, helps partner countries fight malaria, tuberculosis and HIV by financing procurement of medicines for the three conditions. ${ }^{20} \mathrm{It}$ maintains a public list of products that may be procured with its funds. The Global Fund does not conduct its own quality review of ARVs. Rather, an ARV may be added to the Global Fund's public list in three ways: 1) through inclusion on the WHO/PQP list of prequalified medicines; 2) through one-way reliance on registration with an SRA or 3) through approval by WHO's Expert Review Panel. ${ }^{41}$ The WHO's Expert Review Panel is used to review and authorise drugs that are of interest but not 
yet approved by WHO or an SRA for Global Fund use for a limited time. ${ }^{4}$ Thus, Global Fund's reliance on the USFDA registration can be both direct from the USFDA's list of PEPFAR drugs, and indirect from the WHO/PQP list, which includes the USFDA-registered drugs that were added to the WHO/PQP list through one-way reliance.

Since its debut in 2004, the PEPFAR programme has, by general acknowledgement, had a tremendous impact on global HIV care, but the impact of one-way reliance on the USFDA-registered products has not been closely examined. ${ }^{1222}$ The goal of this study is to help the USFDA better understand how its ARV registrations are being used by the global community and how to improve on the existing collaborations. In this analysis, we assess the number of ARV products made available through WHO/PQP and the Global Fund under direct or indirect one-way reliance based on the USFDA registrations.

\section{METHODS}

We conducted a cross-sectional study to determine uptake by WHO/PQP and the Global Fund of the USFDA-registered ARVs under the PEPFAR process, either through direct or indirect reliance. A 'product' for the purposes of this analysis is an ARV treatment option that is produced by a unique manufacturer, at a unique manufacturing plant, has a specific strength and dosage form and is either a single-active ingredient drug, a fixed-dose combination or a copackaged drug. This definition of 'product' is based on the factors used to list an ARV product on the formularies for all three entities. ${ }^{11} 1821$

\section{Sampling approach, data sources, extraction and quality check}

The study only included ARVs that were in active status with the USFDA (either tentatively or fully approved), as of 20 March 2017 (see online supplementary table 1). The analyses excluded products that had been registered and since withdrawn by the manufacturer or rescinded by the USFDA (see online supplementary table 2). Analysis of ARVs on the WHO/PQP list of ARVs and the Global Fund's procurement list was limited to manufacturers that had registered ARVs with the USFDA under the PEPFAR process and did not include those listed by WHO and the Global Fund under other mechanisms.

Data for the USFDA-registered ARVs under the PEPFAR process were collected using two internal USFDA databases (Document Archiving, Reporting and Regulatory Tracking System and the CDER Informatics Platform). WHO and Global Fund data sources used in this study were current as of $20 \mathrm{March} 2017$. The WHO/PQP list of ARVs was collected from WHO/PQP's public database, which was then matched with the USFDA's using drug characteristics, described below (see online supplementary tables 1 and 3). ${ }^{18}$ Similarly, all products on the Global Fund's public list of ARV Pharmaceutical Products (V.132) were collected and matched with the USFDA-registered drugs. ${ }^{21}$ All preferred first-line therapy options listed in the WHO 2016 HIV treatment guidelines were included and matched to the USFDA-registered products. ${ }^{23}$

Data from all sources were extracted and quality checked for accuracy through manual and automated checks to find inconsistent or mismatched information.

\section{Data standardisation and analysis}

The USFDA-registered ARV products that were available for PEPFAR procurement at the time of the study (ie, in fully approved or tentatively approved status) were used as the comparator against the $\mathrm{WHO} / \mathrm{PQP}$ and Global Fund lists. To facilitate comparison across the three data sets used in this study, we standardised, according to the convention listed in the online supplementary section I the following five drug attributes: name, strength, dosage form, manufacturer name and manufacturing site. An ARV product was considered to be matched across the three data sets if the five listed attributes were identical.

We conducted four primary analyses: 1) percentage of the USFDA-registered products also listed on the WHO/ PQP and/or Global Fund lists; 2) temporal analysis to determine the time elapsed between initial positive USFDA action date (tentative approval or full approval) and the WHO prequalification date for products reviewed by both WHO and the USFDA; 3) an overlap analysis, to determine potential for simultaneous reviews, of WHO full regulatory prequalification within 180 days before or after the first positive action by the USFDA and 4) the percentage of the USFDA-registered ARVs that were supportive of preferred first-line therapies for HIV according to the WHO guidelines, and the uptake of those first-line USFDA-registered products by WHO and the Global Fund. ${ }^{24-26}$ A product was considered 'supportive' of WHO's preferred first-line option if it could, either by itself or in combination with other ARVs, constitute an HIV therapy option according to those guidelines. Temporal and overlap analyses were not possible for the Global Fund list because the Global Fund does not operate its own registration process. Detailed methods for each analysis are provided in online supplementary section II.

We also conducted relative-risk calculations comparing adult versus paediatric ARVs and fixed-dose/copackaged versus single-active ingredient formulations to determine if there was an association between uptake of certain products by WHO/PQP or the Global Fund (results are listed in online supplementary section III).

Data were analysed using Excel 2010 and Tableau Desktop V.10.1. ${ }^{27} 28$ Relative risk calculations, along with 95\% CIs, were conducted using MedCalc V.16.8 online statistical software. ${ }^{29}$ All percentages and numbers of days were rounded to the nearest whole number for ease of presentation.

\section{RESULTS}

The number of ARVs available for procurement from the USFDA, WHO/PQP and the Global Fund as of 
Table 1 Total number of ARVs registered or listed by WHO/PQP, Global Fund and the USFDA, by quality assurance programme and drug type

\begin{tabular}{|c|c|c|c|c|c|c|c|}
\hline \multirow[b]{2}{*}{ Drug type } & \multicolumn{2}{|c|}{ WHO QA programme*† } & \multicolumn{4}{|c|}{ Global Fund QA standard* } & \multirow{2}{*}{$\begin{array}{l}\text { USFDAf } \\
\text { PEPFAR } \\
\text { process }\end{array}$} \\
\hline & PQP & USFDA & SRA only & $\begin{array}{l}\text { WHO/PQP } \\
\text { and SRA }\end{array}$ & $\begin{array}{l}\text { WHO/PQP } \\
\text { only }\end{array}$ & ERP & \\
\hline Single drug & 72 & 57 & 65 & 53 & 37 & 0 & 131 \\
\hline 2 Drugs FDC & 34 & 26 & 28 & 22 & 14 & 1 & 51 \\
\hline 3 Drugs FDC & 20 & 13 & 12 & 15 & 7 & 0 & 28 \\
\hline Copackaged & 2 & 10 & 11 & 1 & 4 & 0 & 14 \\
\hline Total & 128 & 106 & 116 & 91 & 62 & 1 & 224 \\
\hline Grand total & 234 & & 270 & & & & 224 \\
\hline
\end{tabular}

*Only products from manufacturers who have also submitted applications to the USFDA for the PEPFAR process were included in this study. †Includes products no longer active with the USFDA, which were excluded in subsequent matching analyses.

$\ddagger$ Only includes products that were in active regulatory status with the USFDA on 20 March 2017 . One additional product from analysis was excluded due to inconsistencies in the USFDA database listing.

ERP, expert review panel; FDC, fixed drug combination; PEPFAR, US President's Emergency Plan for AIDS Relief; PQP, Prequalification of Medicines Programme; QA, quality assurance; SRA, stringent regulatory authority; USFDA, US Food and Drug Administration.

20 March 2017 is shown in table 1. Since the beginning of the programme, the USFDA has registered 246 ARVs through the PEPFAR process; 21 of these are no longer available. Of 225 ARVs currently available, we excluded one additional product from analysis due to inconsistencies in the USFDA database listing, yielding a study sample of 224 active products (see online supplementary table 1). Of the 224, $43 \%$ (93) were fixed-dose combinations or copackaged products and $31 \%$ (70) were for treatment of paediatric populations (data not shown).

\section{Uptake of the USFDA-registered drugs by WHO/PQP and the Global Fund}

The online supplementary table 4 shows the overall disposition of the 224 active USFDA-registered products, broken down by the two WHO/PQP and the three Global Fund quality assurance mechanisms used to add ARVs to their respective lists. Figure 1 merges the various quality assurance mechanisms (see online supplementary table 4) used by WHO/PQP and the Global Fund to highlight the number of the USFDA-registered products used or not used by the two programmes. Overall, $91 \%$ $(204 / 224)$ of the USFDA-registered products appeared on either the WHO/PQP or the Global Fund ARV lists, through direct or indirect mechanisms, and were thus available for procurement.

Forty-five per cent $(100 / 224)$ of the USFDA-registered products appeared on the $\mathrm{WHO} / \mathrm{PQP}$ list through direct one-way recognition, of which 33 were for paediatric use and 67 were for adults (see online supplementary figure 1). Of the 124 USFDA-registered products (37 paediatric; 87 adult) not included in the $\mathrm{WHO} / \mathrm{PQP}$ list through one-way recognition, 73 had undergone WHO's own full prequalification review and seven of these had been prequalified by WHO before the USFDA's PEPFAR review process began operation. Excluding these seven ARVs yields 66 products that underwent both WHO's full prequalification and the USFDA registration (see online supplementary table 5 ). It is possible that some of the $66 \mathrm{ARVs}$ were first added to the WHO list through one-way recognition and then subsequently approved through WHO's own review process. However, since both WHO and FDA lists are regularly updated, overwriting previous entries, we were unable to independently determine if and when such overlaps may have occurred.

The Global Fund listed 79\% (178) of the USDFA-registered ARVs through both direct and indirect reliance (figure 1). The Global Fund directly used 95 USFDA-registered products (through the SRA quality assurance mechanism); and indirectly used 83 products through the $\mathrm{WHO} / \mathrm{PQP}$ list.

Of the 51 USFDA-registered ARVs not found on the $\mathrm{WHO} / \mathrm{PQP}$ list (figure 2), $23(45 \%)$ were for paediatric use, accounting for $33 \%(23 / 70)$ of all the USFDA-registered paediatric products. Of the 46 USFDA products not appearing on the Global Fund or WHO/PQP lists, seven were added to the Global Fund list by relying on WHO full prequalification review, but 39 were not on the Global Fund list at all. Among these 39 products, 12 were for paediatric use and 11 were fixed-dose or copackaged products (figure 2 ).

\section{Temporal analysis}

Since December 2004, a total of 66 of 224 products have received both WHO full prequalification and the USFDA registration; $30 \%(20 / 66 ; 5$ paediatric) were prequalified by WHO before the USFDA registration and $70 \%$ (46/66; 7 paediatric) received prequalification by WHO after the USFDA registration had been completed (see online supplementary figure 2 and online supplementary table 5). Figure 3 shows the time elapsed between the USFDA registration and WHO prequalification of ARVs reviewed by both entities, in months, before or after the USFDA registration. The 20 products prequalified by WHO first were done so a median of 250 days 


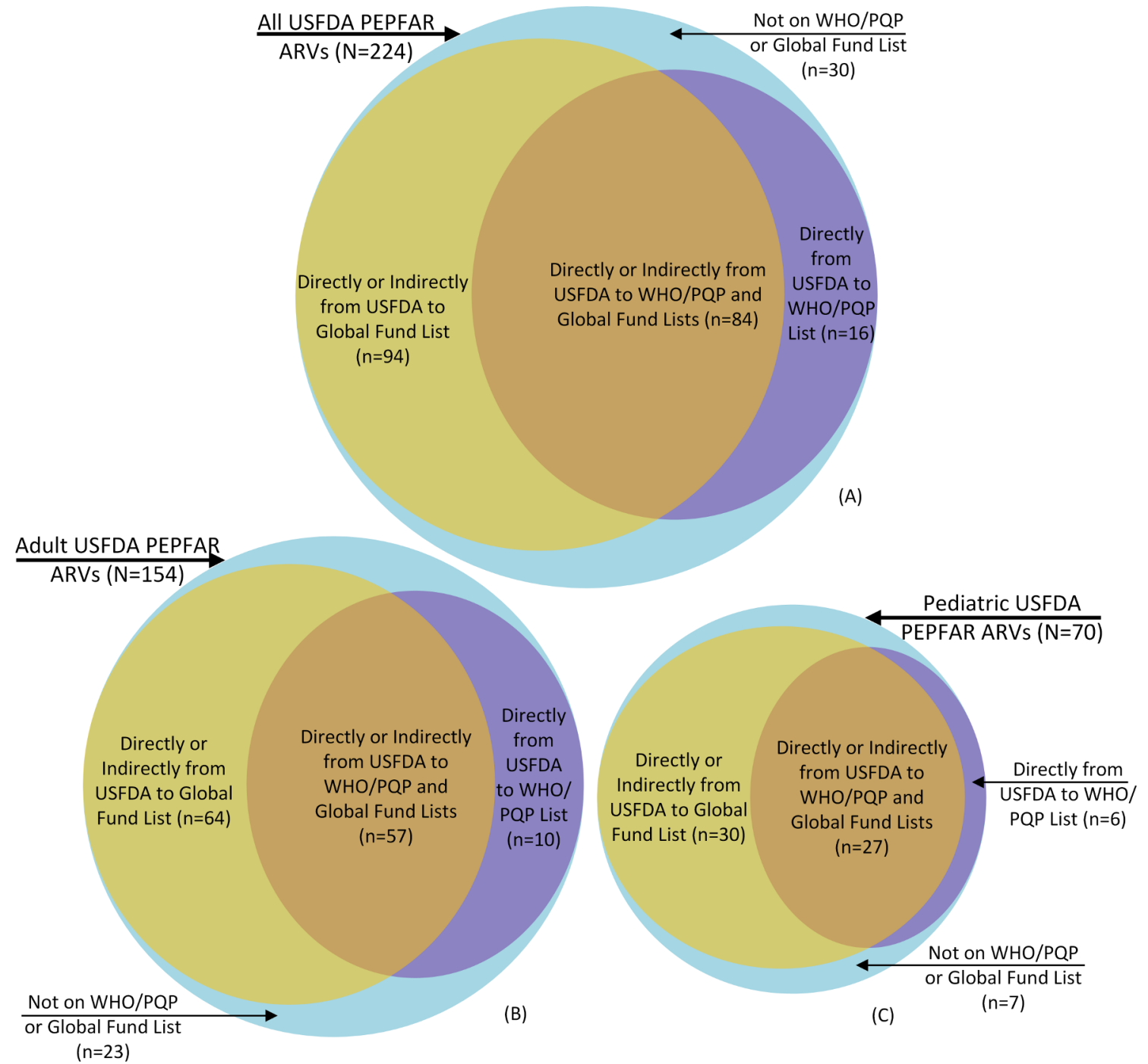

Figure 1 Number of ARVs from the USFDA PEPFAR list adopted by WHO, the Global Fund or both, overall and by population. The figure shows the total number of the USFDA-registered products that could be used to support one-way reliance by WHO and the Global Fund. The smaller circles show the number of the USFDA-registered products used by WHO (purple circle) and the Global Fund (yellow circle) or both (dark orange circle) through one-way reliance to support procurement. The overall figure on 224 products $(A)$ is further described for adult ARVs $(B)$ and paediatric products $(C)$. ARV, antiretroviral; PQP, Prequalification of Medicines Programme; USFDA, US Food and Drug Administration.

(IQR 85-544) before the USFDA registration (figure 3 and online supplementary figure 3 ). The 46 products that first underwent the USFDA registration did so by a median of 559 days (IQR 233-798 days) before WHO prequalification; the seven paediatric products compared with adult drugs received prequalification a median of 110 days after the USFDA registration (see online supplementary table 6 ).

\section{Analysis of overlap of drug dossier review}

Additional analysis of the potential for simultaneous product reviews by the USFDA and the WHO full prequalification programme showed that $18 \mathrm{ARVs}$ were prequalified by WHO within 180 days of the first positive action by the USFDA (see online supplementary figure 2 and online supplementary table 7 ). Ten of the 18 products were registered by $\mathrm{WHO} / \mathrm{PQP}$ after the USFDA registration; these 10 were prequalified a median of 108 days
(IQR 65-126 days) after the USFDA registration. The remaining eight were prequalified a median of 83 days (IQR 21-133 days) before receiving the USFDA registration.

\section{WHO-preferred first-line HIV therapies}

Forty-one per cent $(91 / 224)$ of current USFDA-registered products have the potential to support the 2016 WHO-preferred first-line HIV treatment options (see online supplementary table 8 , figure 4 ). Of the 91 products, 36 (17 adult, 19 paediatric) were adopted by WHO through one-way recognition and 16 products (7 adult, 9 paediatric) were not found on the WHO list (figure 4). The remaining 39 were added to the WHO list using full prequalification review, of which three products that predated USFDA's PEPFAR programme were excluded from further analysis. Of the $36,72 \%$ (26) were WHO prequalified after the USFDA registration (median 


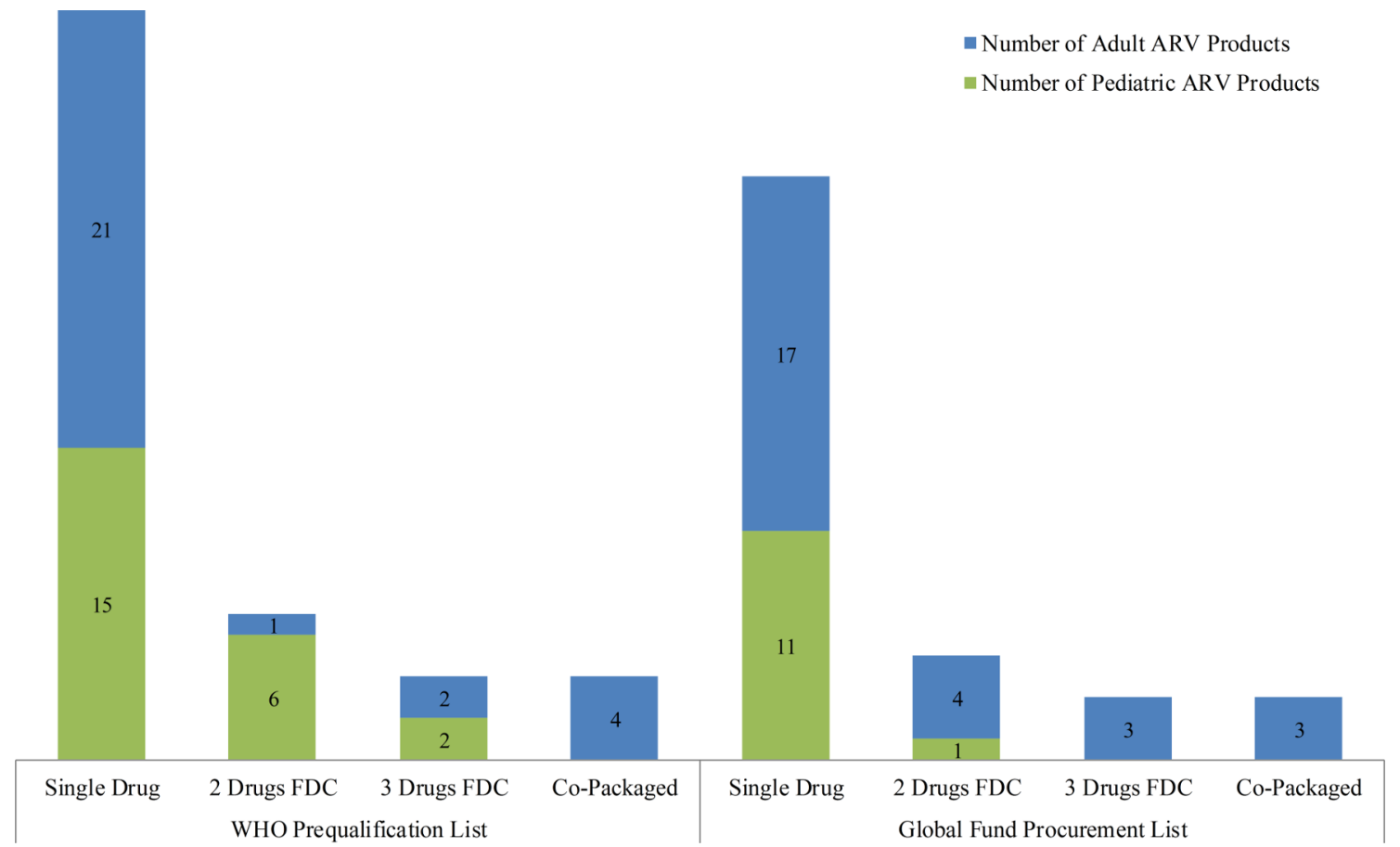

Figure 2 Number and type of the US Food and Drug Administration-registered ARVs not taken up through one-way recognition on WHO/PQP or Global Fund procurement lists, by population. ARV, antiretroviral; FDC, fixed drug combination.

513 days (IQR 294-798 days)); and the remaining 10 were WHO prequalified before the USFDA registration (median 288 days (IQR 81-439 days) (see online supplementary table 9). The Global Fund included on its list, through direct and indirect reliance, $85 \%$ (77/91) of the USFDA-registered products that support WHO-preferred first-line HIV treatment.

\section{DISCUSSION}

The USFDA has registered a wide range of ARV products through its dedicated PEPFAR process, 224 of which were available for procurement by global partners. The registered products include 93 fixed-dose or copackaged products that make treatment regimens simpler by reducing pill burden or frequency, potentially leading to increased adherence. ${ }^{30}$ Seventy of the 224 products were for paediatric patients; this is of particular importance as worldwide there were about 1.8 million children living with HIV in 2015, with 150000 new paediatric infections annually. ${ }^{31}$ Our analysis found that among the 224 USFDA-registered products, there was a significant overlap with the products on the WHO/PQP $(77 \%)$ and the Global Fund quality assurance (83\%) lists used for procurement.

Although WHO and the Global Fund may use ARVs from multiple sources to enhance their respective quality-assured drug list, both use a large number of ARVs that were originally registered by the USFDA for PEPFAR. However, the extent to which the USFDA-registered ARV products were used to augment both the $\mathrm{WHO} / \mathrm{PQP}$ and Global Fund procurement lists varies. WHO and Global Fund used $45 \%$ and $79 \%$ of the USFDA-registered products through one-way recognition, respectively. Although the differential uptake by WHO and the Global Fund cannot be attributed to any single reason, the existence of the WHO-managed prequalification of medicines programme, in which WHO reviews and registers medicines, likely plays a significant role. ${ }^{17}$ In contrast, the Global Fund does not operate a similar process, instead uses an expert panel for risk assessment of products not yet registered by an SRA or WHO/PQP. This panel, however, is only used for temporary or short-term procurement of ARVs until they are quality-assured by an SRA or WHO/PQP. ${ }^{4}$

In general, entities supporting procurement may strive to attain a balanced list of products that allow purchasing of a wide array of ARVs to meet the needs of their respective partner countries and programmes. Such needs may include: 1) ensuring an adequate number of manufacturers for the same product to meet demand and prevent shortages; 2) availability of commonly used products, even if certain ARVs are no longer the preferred options; 3) ensuring healthy competition from multiple manufacturers to lower procurement costs; and 4) use of product formulations that support efficiency in procurement, storage and distribution (ie, heat-stable, long shelf-life or non-refrigerated products).$^{32}$

Another reason for differential uptake by WHO could be that the manufacturer of the product must provide WHO with permission to add the USFDA-registered products to the WHO list under its 'alternative listing procedure'. ${ }^{16}$ We did not study how often, if ever, such permission is denied. Furthermore, since 2012, the manufacturers must proactively approach $\mathrm{WHO} / \mathrm{PQP}$ to get 
A

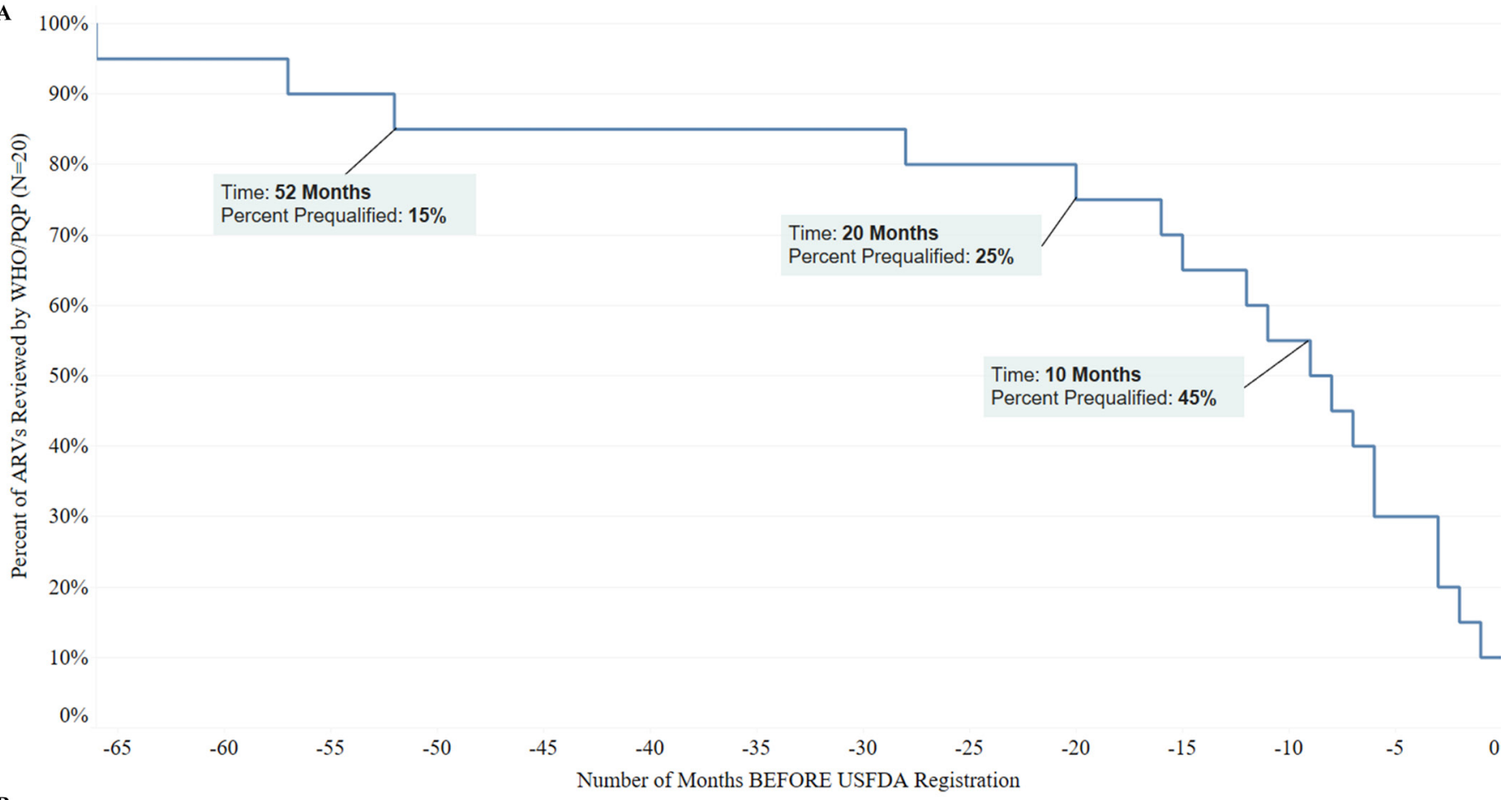

B

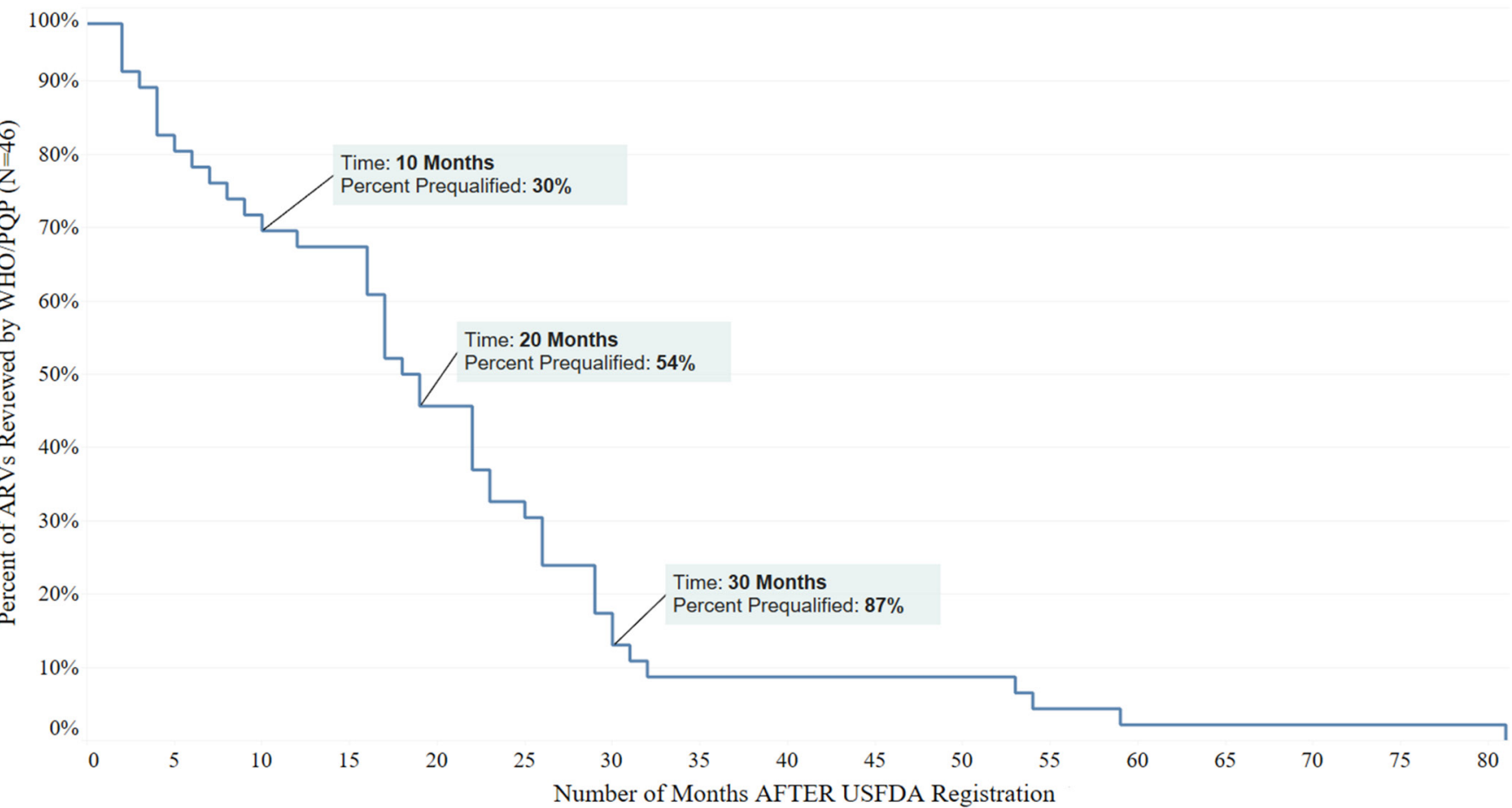

Figure 3 Per cent and timeline of ARV products reviewed by WHO/PQP before and after the USFDA registration. The graphs indicate the per cent of the applications reviewed and approved by WHO/PQP either before $(n=20)$ or after $(n=46)$ the USFDA registration. Negative months indicate time before the USFDA registration. Panel (A) shows that of the 20 products, about $45 \%, 25 \%$ and $15 \%$ of the products were prequalified 10, 20 and 52 months before the USFDA, respectively. Panel (B) shows that out of the 46 products, $30 \%, 54 \%$ and $87 \%$ were approved by WHO at 10,20 and 30 months after the USFDA registration, respectively. ARV, antiretroviral; PQP, Prequalification of Medicines Programme; USFDA, US Food and Drug Administration.

their USFDA-registered ARVs listed on the WHO/PQP list. Prior to 2012, WHO asked manufacturers to consider listing their products on the PQP list via one-way reliance, potentially resulting in higher one-way adoption. Even if a manufacturer wishes to list a USFDA-registered drug on the PQP list via one-way reliance, WHO's full prequalification review will only stop if requested by the applicant. As such, some USFDA-registered ARVs initially adopted 
- Number of USFDA Registered ARVs Placed on WHO List through One-Way Recognition $(\mathrm{N}=36)$

n Number of USFDA Registered ARVs Not Placed on WHO List $(\mathrm{N}=16)$

number of USFDA Registered ARVs Added with Full WHO/PQP Review $(\mathrm{N}=39)$

n Total Number of USFDA Registered ARVs with potential to support WHO First-Line Treatments $(\mathrm{N}=91)$
53

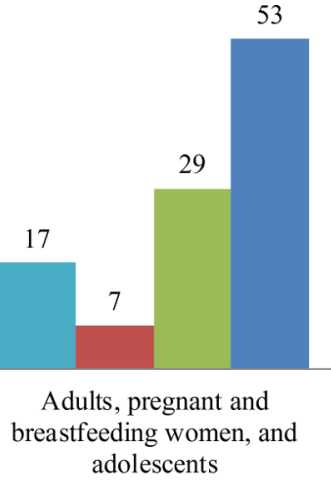

Population Groups for Preferred First-Line WHO Treatment Options

Figure 4 WHO-preferred first-line HIV treatment options supported by the USFDA PEPFAR ARVs and their availability on the WHO/PQP list. ARV, antiretroviral; PQP, Prequalification of Medicines Programme; USFDA, US Food and Drug Administration.

by WHO through one-way reliance, may eventually attain WHO full prequalification if a full dossier is submitted to $\mathrm{WHO}$, which would then serve as the quality assurance mechanism. Furthermore, completing WHO's full prequalification programme is necessary if the manufacturer wishes to have its product under WHO oversight (variations, reinspections, requalification) and register its product in countries via the collaborative registration procedure, which would not be possible for the USDFAonly registered products. Additionally, $\mathrm{WHO} / \mathrm{PQP}$ only reviews or lists products that are the subject of a specific request through a process called invitation for expression of interest. ${ }^{33}$ An invitation is only issued for products meeting key requirements, such as for a product recommended by current WHO treatment guidelines. ${ }^{17}$ As such, some USFDA-registered ARVs may not be the subject of WHO invitation and consequently will not be included in the PQP list; we did not study how many USFDA-registered drugs were not issued WHO invitations. Determination of the exact reasons and mechanisms via which some products are included or excluded from WHO or the Global Fund lists is beyond the scope of this study.

Our analysis also found that a substantial number of the USFDA-registered products (91 of the 221 total; 38 for paediatric use) have the potential to support the 2016 WHO HIV treatment guidelines. Such products are of particular importance as the guidelines may be used by low-income and middle-income countries and clinicians to provide HIV/AIDs care, as well as by policy makers and global implementing agencies to make procurement decisions. ${ }^{23-2634}$ However, 27 of the 91 USFDA-registered ARVs that support treatment guidelines were not found in the WHO/PQP list (16 ARVs) or the Global Fund list (11 ARVs). The reasons for the exclusions are unknown but are presumably related to the previously discussed complexities.

Although some USFDA-registered ARVs were not found on the WHO and Global Fund lists, their impact on clinical care may vary. For example, 14 of the 23 paediatric products not on the WHO list contain stavudine or didanosine, two ARVs that are no longer mainstays of HIV therapy due to high toxicity, as such no longer preferred first-line options on WHO guidelines. ${ }^{23} 35$ The remaining nine products, however, do have the potential to support paediatric treatment according to the WHO HIV treatment guidelines. We did not study whether similar products from other manufacturers with preferable formulations are available on the WHO list or are under review by PQP. Among the 12 paediatric products not on the Global Fund list, seven do not support current WHO treatment options. We did not determine whether the omitted products on the WHO/PQP or the Global Fund lists are available from alternative sources.

We found that $55 \%$ of the USFDA-registered products were not used for one-way recognition by WHO. Another $29 \%$ of the USFDA-registered ARVs had undergone both the USFDA registration and full WHO prequalification review; some of these received WHO prequalification a year-and-a-half after the USFDA registration. Furthermore, 18 products received both the USFDA registration and WHO prequalification within 6 months of each other. This indicates that 1) some of the same products are being reviewed by both entities and 2) that some of the reviews are simultaneous or overlap closely. We did not study when ARVs were submitted to $\mathrm{WHO} / \mathrm{PQP}$, if the manufacturer provided $\mathrm{WHO}$ with the appropriate permissions, whether WHO review was impeded by resource constraints or if WHO had specific safety, efficacy or quality concerns not shared by the USFDA, all of which could provide context for the overlap observed.

Furthermore, manufacturers may be submitting applications simultaneously to both entities to 1 ) maximise their chances of success and 2) to avail themselves of advantages offered by having both the USFDA registration and full prequalification by WHO. While the USFDA registration allows manufacturers to sell their products for PEPFAR and Global Fund procurement, full WHO prequalification has the added advantage of allowing them to participate in WHO's Collaborative Registration Procedure (WHO/CRP). ${ }^{2}$ The WHO/CRP allows manufacturers with WHO-prequalified products to accelerate registration in countries where the ARVs will ultimately be used; this is accomplished through information sharing between the $\mathrm{WHO} / \mathrm{PQP}$ and national 
medicines regulatory authorities. ${ }^{2}$ ARVs registered solely with the USFDA are not eligible for participation in the WHO/CRP.

Another reason for duplicative reviews could be that until 1 October 2012, review of generic drug applications by the USFDA was free, as was review of all applications by WHO/PQP until 1 September $2013 .{ }^{35} 36$ On the other hand, new drug applications (for innovative combinations, formulations and copacking) submitted to the USFDA for PEPFAR, have always been and continue to be free to the applicants. Absence of applications fees likely provided a no-cost venture to the applicants, resulting in concurrent submissions. Any of the above might explain our observations for duplicate or simultaneous reviews.

Lack of real-time coordination among the USFDA, $\mathrm{WHO} / \mathrm{PQP}$ and manufacturers could cause delays in the addition of clinically relevant and the USFDA-registered ARVs to the WHO list. For example, a new, easy-to-use, heat-stable formulation of lopinavir/ritonavir in a pellet form, a major step forward for treatment of infants and young children, was registered by the USFDA in May 2015. ${ }^{3738}$ However, at the time of this writing, the product was not on the WHO list. Timely listing of clinically relevant products may help procurement entities better assess the array of available ARVs and allocate resources accordingly. As discussed above, the drug manufacturer could ask WHO to add its ARV to the PQP list; however, a coordinated approach between the three actors (and possibly other SRAs), could lead to faster, real-time harmonisation of the USFDA and WHO lists. This, in turn, could provide procurement entities and other global HIV programmes with an up-to-date comprehensive list of high-quality ARVs.

An overarching effort for early and frequent collaboration between WHO, the USFDA and the manufacturers could help reduce the impact of dual submissions, improve listing of necessary USFDA-registered ARVs on the WHO list, and increase access. Under an existing confidentiality agreement, the USFDA, with standing permission from the applicant, may share "confidential product and/or commercial information (and) trade secret information' on each registered PEPFAR ARV to support one-way recognition. ${ }^{39}$ The agreement is a broad commitment between the USFDA and the WHO/PQP for the purposes of 'undertaking discussions aimed at coordinating and facilitating (USFDA's) regulatory activities and (WHO's) prequalification activities'. ${ }^{39}$ Using this mechanism, the USFDA, with applicant's permission, may share the complete drug dossier assessments with WHO/ $\mathrm{PQP}$ to allow for an abbreviated review by PQP to avoid duplication of effort. Once prequalified, the ARVs may then be considered under the WHO/CRP for accelerated approval in participating countries. ${ }^{2}$ All entities should explore whether a more streamlined process to share real-time information on product submissions between WHO and the USFDA drug review centres under these pre-existing channels may help save resources. ${ }^{39}$
Any such collaboration would be beneficial to all stakeholders: the USFDA could improve the usability of its registered ARVs by making them available to a wider group of countries faster; WHO/PQP may be able to save resources on review of ARVs and reallocate them to other priorities; procurement mechanisms could be made aware of critically needed and quality-assured ARVs in a timely manner; manufacturers could benefit from increased demand and better forecasting and ultimately, more patients could receive life-saving drugs earlier.

This study has several limitations. First, the USFDA, WHO/PQP and Global Fund lists are updated frequently with new additions or removals, which may increase or decrease matching, potentially affecting the precise matching of products but is unlikely to significantly affect the overall findings. Second, we did not consider ARVs that are on the WHO/PQP and Global Fund lists but have not been registered by the USFDA. Third, for the overall matching and non-matching analysis, all active USFDA products were analysed without consideration of current clinical relevance of some of the ARVs. We did, however, conduct a focused analysis of the USFDA-registered ARVs supportive of preferred first-line options per 2016 WHO treatment guidelines. Fourth, the Global Fund may add non-PEPFAR drugs registered by the USFDA (while WHO's additions based on its alternative listing procedure has primarily relied on PEPFAR ARVs); we did not study this, as the ARVs reviewed under the dedicated PEPFAR process were the primary focus of this analysis. However, the overall impact of the non-PEPFAR process drugs is expected to be limited, as such drugs are innovator ARVs, which are significantly more expensive than the USFDA PEPFAR process drugs and thus less likely to be procured in resource-constrained settings. ${ }^{12}$ Fifth, this study did not analyse if the drugs are being produced by the manufacturer or if they are being actively procured for use, but only if they were eligible for procurement. Sixth, our temporal analysis does not assess application review times by the USFDA or by WHO/PQP; we only studied the time elapsed between registration actions of WHO and the USDFA. Seventh, this study did not consider if the USFDA-registered ARVs are authorised for use in all countries that could ultimately use the products.

\section{CONCLUSION}

To our knowledge, this is the first study to examine the impact of the USFDA-registered ARVs on WHO and the Global Fund through one-way recognition. We found that the USFDA's work on ARV products has supported and continues to support the global HIV fight by making products available through one-way reliance by WHO and the Global Fund. This novel approach by WHO and the Global Fund has enhanced efficiency in regulatory review and quality assurance. The study also found a significant overlap in timing between products submitted to both WHO and the USFDA for review, and the potential for delayed listing of quality assured 
ARVs on WHO's comprehensive list. Improved efforts to share real-time information and promote collaboration between the USFDA, WHO and the manufacturers may help streamline product reviews and minimise duplication of efforts.

\section{Author affiliations}

${ }^{1}$ Office of Public Health Strategy and Analysis, Office of the Commissioner, US Food and Drug Administration, Silver Spring, MD, USA

${ }^{2}$ Office of Pharmaceutical Quality, Center for Drug Evaluation and Research, US Food and Drug Administration, Silver Spring, MD, USA

${ }^{3}$ Office of Generic Drugs, Center for Drug Evaluation and Research, US Food and Drug Administration, Silver Spring, MD, USA

${ }^{4}$ Office of New Drugs, Center for Drug Evaluation and Research, US Food and Drug Administration, Silver Spring, MD, USA

${ }^{5}$ Office of International Programs, Office of the Commissioner, US Food and Drug Administration, Silver Spring, MD, USA

Acknowledgements The authors would like to thank Dr Matthias Stahl at WHO Prequalification of Medicines Programme, who reviewed the draft manuscript and helped contextualise the findings in consideration of WHO policies and practices; and Ms. Lisa Rovin and Ms. Anam Chaudhry from the US Food and Drug Administration, for their thoughtful and in-depth review of manuscript.

Contributors HSC conceived the study, developed the methodology, designed the data collection instrument, oversaw data collection, analysed the results and played a leading role in writing the manuscript. PC and RP collected and verified data for US Food and Drug Administration drugs. PC, RP, JSM, MS and MLV reviewed, substantially edited and revised the manuscript. PGL helped refine the methodology, reviewed and substantially edited the manuscript and provided overall direction for the study. All authors contributed to discussion of the findings and policy implications.

Funding The authors have not declared a specific grant for this research from any funding agency in the public, commercial or not-for-profit sectors.

Disclaimer The views expressed in this paper are those of the authors and not necessarily those of the US Food and Drug Administration. PGL is no longer with the US Food and Drug Administration.

Competing interests None declared.

Patient consent Not required.

Provenance and peer review Not commissioned; externally peer reviewed.

Data sharing statement No additional data are available. All study data are provided in the supplement

Open Access This is an Open Access article distributed in accordance with the Creative Commons Attribution Non Commercial (CC BY-NC 4.0) license, which permits others to distribute, remix, adapt, build upon this work non-commercially, and license their derivative works on different terms, provided the original work is properly cited and the use is non-commercial. See: http://creativecommons.org/ licenses/by-nc/4.0/

(c) Article author(s) (or their employer(s) unless otherwise stated in the text of the article) 2018. All rights reserved. No commercial use is permitted unless otherwise expressly granted.

\section{REFERENCES}

1. Chahal HS, Murray JS, Shimer M, et al. The US Food and Drug Administration's tentative approval process and the global fight against HIV. Journal of the International AIDS Society 2017:20:e25019.

2. World Health Organization. WHO Prequalification of Medicines Programme - WHO launches the PQP Collaborative Registration Procedure. WHO Drug Information. Geneva, Switzerland: World Health Organization, 2013.

3. Preston $\mathrm{C}$, Chahal HS, Porrás A, et al. Regionalization as an approach to regulatory systems strengthening: a case study in CARICOM member states. Rev Panam Salud Publica 2016;39:262-8.

4. The Global Fund. Quality Assurance: Medicines. 2016 http://www. theglobalfund.org/en/sourcing/qa/medicines/ (accessed 18 Aug 2016).
5. World Health Organization. Model quality assurance system for procurement agencies. Geneva, Switzerland: WHO Technical Report, 2014.

6. Institute of Medicine. Riviere JE, Buckley GJ, Ensuring Safe Foods and Medical Products Through Stronger Regulatory Systems Abroad. Washington (DC), 2012.

7. Institute of Medicine. Buckley GJ, Gostin LO, Countering the Problem of Falsified and Substandard Drugs. Washington (DC), 2013.

8. Chahal HS, Kashfipour F, Susko M, et al. Establishing a regulatory value chain model: An innovative approach to strengthening medicines regulatory systems in resource-constrained settings. Rev Panam Salud Publica 2016;39:299-305.

9. 't Hoen EF, Hogerzeil HV, Quick JD, et al. A quiet revolution in global public health: The World Health Organization's Prequalification of Medicines Programme. J Public Health Policy 2014;35:137-61.

10. Venkatesh KK, Mayer KH, Carpenter CC. Low-cost generic drugs under the President's Emergency Plan for AIDS Relief drove down treatment cost; more are needed. Health Aff 2012;31:1429-38.

11. US Food and Drug Administration. Approved and Tentatively Approved Antiretrovirals in Association with the President's Emergency Plan. 2016 http://www.fda.gov/InternationalPrograms/ PEPFAR/ucm119231.htm (accessed 1 Sep 2016).

12. Holmes $C B$, Coggin W, Jamieson $D$, et al. Use of generic antiretroviral agents and cost savings in PEPFAR treatment programs. JAMA 2010;304:313-20.

13. Padian NS, Holmes CB, McCoy SI, et al. Implementation science for the US President's Emergency Plan for AIDS Relief (PEPFAR). J Acquir Immune Defic Syndr 2011;56:199-203.

14. Lantos T, Henry J. Hyde United States Global Leadership Against HIVIAIDS, Tuberculosis, and Malaria Reauthorization Act of 2008, in 22 U.S.C. 7601. United States, 2008.

15. World Health Organization. Collaborative Registration of SRAApproved Medicines - pilot. 2016 http://apps.who.int/prequal/info applicants/collaborative_registration_SRA.htm\#l (accessed 18 Aug 2016).

16. World Health Organization. General information on the WHO List of Prequalified Medicinal Products: Alternative listing procedures. 2016 http://apps.who.int/prequal/info_general/notes_registry.htm\#3 (cited 2016 August 18)

17. World Health Organization. Prequalification of medicines by WHO. 2016 http://www.who.int/mediacentre/factsheets/fs278/en/ (Accessed 18 Aug 2016).

18. World Health Organization. WHO List of Prequalified Medicinal Products. 2016 http://apps.who.int/prequal/info_general/notes.htm (Accessed 13 May 2016).

19. Medicines Patent Pool. Licences in the MPP. 2018 https:// medicinespatentpool.org/what-we-do/global-licence-overview/ licences-in-the-mpp/ (accessed 16 Jan 2018).

20. The Global Fund. Global Fund Overview. 2016 http://www. theglobalfund.org/en/overview/ (accessed 1 Sep 2016).

21. The Global Fund. List of ARV Pharmaceutical Products classified according to the Global Fund Quality Assurance Policy (Version 132), 2016.

22. US President's Emergency Plan for AIDS Relief (PEPFAR). Fact Sheet - 2015 PEPFAR Latest Results. 2015 http://www.pepfar.gov/ documents/organization/250324.pdf (accessed 15 Jun 2016).

23. World Health Organization. Consolidated guidelines on the use of antiretroviral drugs for treating and preventing HIV infectionRecommendations for a public health approach. Second edition. Geneva, Switzerland: World Health Organization, 2016:480

24. Holmes $\mathrm{C}$, Pillay $\mathrm{Y}$, Mwango A, et al. Health systems implications of the 2013 WHO consolidated antiretroviral guidelines and strategies for successful implementation. AIDS 2014;28(Suppl 2):S231-39.

25. Hsieh AC, Mburu G, Garner AB, et al. Community and service provider views to inform the 2013 WHO consolidated antiretroviral guidelines: key findings and lessons learnt. AIDS 2014;28(Suppl 2):S205-16.

26. Ishikawa N, Shimbo T, Miyano S, et al. Health outcomes and cost impact of the new WHO 2013 guidelines on prevention of mother-tochild transmission of HIV in Zambia. PLoS One 2014;9:e90991.

27. Microsoft. Microsoft Excel. Redmond, WA. 2010.

28. Tableau Software. Tableau Desktop. Seatle, WA. 2016.

29. MedCalc. Relative risk calculator (Version 16.8), 2016. https://www. medcalc.org/calc/relative_risk.php. (accessed 24 Aug 2016).

30. Airoldi M, Zaccarelli M, Bisi L, et al. One-pill once-a-day HAART: a simplification strategy that improves adherence and quality of life of HIV-infected subjects. Patient Prefer Adherence 2010;4:115-25.

31. Joint United Nations Programme on HIV/AIDS (UNAIDS). AIDS by the numbers - Ending the AIDS epidemic by 2030 as part of the 
sustainable development goals. Geneva, Switzerland: UNAIDS-Joint United Nations Programme on HIV/AIDS, 2016:28.

32. Pan American Health Organization. A Practical Guide For Procurement Planning And Management Of Strategic Public Health Supplies. Washington (DC): Pan American Health Organization, 2006:67.

33. World Health Organization. WHO Expert Committee on Specifications for Pharmaceutical Preparations - Forty-Third Report, in WHO Technical Report Series. Geneva, Switzerland: World Health Organization, 2009.

34. Nelson LJ, Beusenberg M, Habiyambere V, et al. Adoption of national recommendations related to use of antiretroviral therapy before and shortly following the launch of the 2013 WHO consolidated guidelines. AIDS 2014;28(Suppl 2):S217-24.

35. World Health Organization. Consolidated guidelines on the use of antiretroviral drugs for treating and preventing HIV infection -
Supplement to Chapter 9: Phasing out stavudine: Progress and Challenges. Geneva, Switzerland: World Health Organization, 2013:17.

36. US Food and Drug Administration. Generic Drug User Fee Amendments of 2012 (GDUFA). $2016 \mathrm{http}: / / \mathrm{www} . f d a . g o v /$ Forlndustry/UserFees/GenericDrugUserFees/default.htm (accessed 27 Jun 2016).

37. World Health Organization. The Interagency Task Team (IATT), and United Nations Children's Fund, Fact Sheet on Lopinavir and Ritonavir (LPV/R) Oral Pellets. 2015.

38. US Food and Drug Administration. Tentative Approval-NDA 205425.

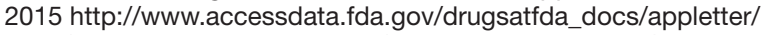
2015/2054250rig1s000TAltr.pdf (accessed 31 Aug 2016).

39. US Food and Drug Administration and World Health Organization. FDA-WHO/SM, Mutual Confidentiality Commitment. $2005 \mathrm{http} / / /$ www.fda.gov/InternationalPrograms/Agreements/ConfidentialityC ommitments/ucm095342.htm (accessed 18 Aug 2016). 